\title{
Backscatter Electron Imaging Verses "Wein-type" Filtered Secondary Electron Imaging of Thick Methacrylate Sections of Tissues in a Field Emission Scanning Electron Microscope (FESEM)
}

\author{
C.A. Ackerley*, A. Tilups*, C. Nielsen** and M.A. Coy** \\ *Division of Pathology, Hospital for Sick Children, Toronto, ON \\ **JEOL USA, Peabody, MA
}

A limiting factor in the study of sections of cells and tissues using electron microscopy has been the sample size. Specimens must be extremely small when being processed (usually no bigger than 1 $\mathrm{mm}^{3}$ ) in order to insure good fixation and embedding. Once embedded the sample must be sectioned. Typically, $0.5-1 \mu \mathrm{m}$ sections of epoxy embedded materials are often first examined with a light microscope and the block trimmed to a region of interest. Ultrathin sections are then prepared prior to examination in the transmission electron microscope (TEM). As a result sample size is limited leading to potential interpretive biases. Alternatively, these epoxy embedded light microscope sections can be viewed using backscatter electron imaging (BEI) with either a Scanning Electron Microscope (SEM) at resolutions approaching 100nm or a FESEM at resolutions similar to those obtained in the TEM with ultrathin sections [1,2]. Because of the physical size limitations imposed by this kind of preparation sample bias remains an issue however using methacrylate resins, sections as large as $1 \mathrm{~cm}^{2}$ and $2 \mu \mathrm{m}$ thick can be cut and examined.

Methacrylate sections were examined using the annular BEI detector. For comparison specimens were also examined using a "Wein-type" filter. This consisted of a pre aperture control lens anode in a semi-in lens FESEM with an external power supply. Z-contrast was generated by applying a negative voltage to the anode.

All animals were perfused with a solution of $1 \%$ glutaraldehyde and $4 \%$ formaldehyde in phosphate buffer and the tissues chopped into pieces approximately $1 \times 1 \times 0.2 \mathrm{~cm}$. Following overnight fixation the samples were washed in buffer, dehydrated through a graded series of ethanol to $100 \%$ at room temperature and infiltrated and embedded at $4^{\circ} \mathrm{C}$. Tissues were allowed to polymerize at room temperature in embedding molds. Blocks were sectioned on a Sorval JB-4 microtome at a thickness of $2 \mu \mathrm{m}$ and mounted on glass coverslips. Sections were immersed in aqueous $2 \% \mathrm{OsO} 4$ followed by a saturated solution of thiocarbohydrazide. The sections were again treated with OsO4 and stained with uranyl acetate and lead citrate. Prior to examination in the FESEM samples were rendered conductive with either a thin coat of evaporated carbon or sputter coated chromium.

Specimen examination using the BEI detector required more beam current as well as a higher probe current when compared to the filtered images. As methacrylate resins are extremely electron beam sensitive it was impossible to obtain images from the same area as electron beam damage had occurred therefore comparable areas were examined. Ultrastructure was distinguishable using BEI however structures such as the mitochondria lacked any internal detail and ribosomes in the rough endoplasmic reticulum were difficult to discriminate(figure A). This was probably due to Z-contrast originating within the sections. Filtered images yielded superior ultrastructure. Both ribosomes and the cisternae of the rough endoplasmic reticulum were easily identified as were some of the mitochondrial membranes(figure B). 
The combination of larger tissues for processing, larger sections that don't require thin sectioning, unobscurred low magnification imaging of large fields and the high resolution of the FESEM filter holds great promise for studying any type of biological sample. Further work is being done using other staining protocols and embeddents. Ultimately methods will be devised for cytochemical and immunocytochemical labeling of sections. Correlative studies using both light and electron microscopy will be done on the same section. Although the detail is not as good as that obtained with TEM the sample size yields ultrastructural information from a much larger area.

\section{References}

[1] M.D. Mckee et al., J Bone Miner Res, 6 (9) (1991) 937.

[2] A. Nanci et al., J Histochem Cytochem 38 (3) (1990) 403.
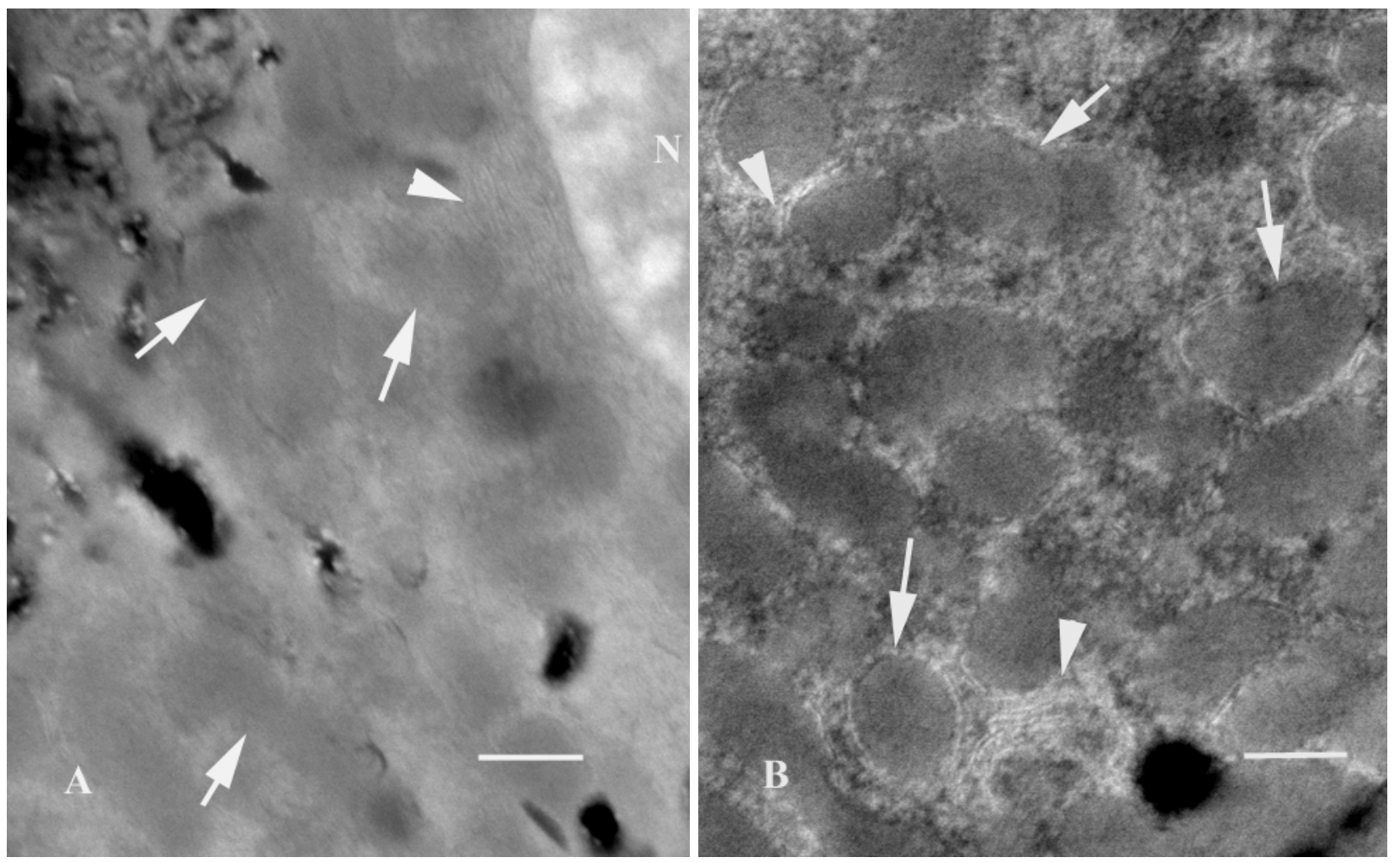

Figure A. BEI of a methacrylate section of mouse liver. Mitochondria (arrows), RER (arrowhead) and a nucleus $(\mathrm{N})$ are distinguishable.

Figure B. Filtered image of the same preparation. Note the superior ultrastructural detail. Ribosomes and ER cisternae are easily distiguished (arrowheads) as are the mitochondria (arrows)

All bars equal $0.5 \mu \mathrm{m}$. 